ANNALES

UNIVERSITATIS MARIAE CURIE-SKŁODOWSKA

LUBLIN - POLONIA

VOL. LXIX, 1-2

SECTIO AA

2014

\title{
Perchloric acid catalyzed condensation of amine and aldehydes: Synthesis and antibacterial activities of some aryl $(E)$-imines
}

\author{
P. Mayavel, K. Thirumurthy, S. Dineshkumar \\ and G. Thirunarayanan ${ }^{\mathrm{a}}$ \\ Department of Chemistry, Annamalai University, \\ Annamalainagar-608002, India. \\ ae-mail:drgtnarayanan@gmail.com
}

\begin{abstract}
A series of Schiff's bases (aryl $E$-imines) have been derived from the perchloric acid catalyzed condensation of aryl amines and substituted benzaldehydes. The yield of the Schiff's bases are more than $80 \%$. The synthesized Schiff's bases are characterized by their physical constants, analytical and spectroscopical data. The antibacterial activities of these Schiff's bases have been studied using Bauer-Kirby method.
\end{abstract}

Keywords: Schiff's bases, Perchloric acid, IR and NMR spectra, Antibacterial activities.

\section{INTRODUCTION}

Schiff's bases were first synthesized by Schiff in 1864. Schiff's bases named after Hugo Schiff's formed by the bimolecular condensation products of primary amine with carbonyl compounds. Schiff's bases are characterized by the $-\mathrm{N}=\mathrm{CH}-$ imine group which find important in elucidating the mechanism of transamination and racemization reaction of biological system [1,2]. Many reagents have been used for the synthesis of optically active imines like Lewis acids [3], molecular sieves in ionic 
liquids [4], solid super acid K-10 montmorillonite [5], Tandam catalyst [6], $\mathrm{MnO}_{2}$ [7], $\mathrm{CaO}$ [8], $\mathrm{ZnCl}_{2}$ [9], $\mathrm{MgSO}_{4}$-PPTS [10], alumina [11], $\mathrm{P}_{2} \mathrm{O}_{5}-\mathrm{SiO}_{2}$ [12], infrared [13], ultrasound radiation [14] and fly-ash: $\mathrm{H}_{2} \mathrm{SO}_{4}$ with microwave irradiation. These catalysts were used for the synthesis of chiral imines by condensation of amines $[15,16]$, with carbonyl compounds [5, 6, 17], alcohols [18] and acid chlorides [3,19]. The imine starting materials and important intermediates were used for the synthesis of pharmacologically active heterocycles including triazoles, trizolones [20-22]. Optically active imine derivatives possess biological activities such as antimicrobial [23], anticancer [24], antiplasmodic-antihypoxic [25], antitubularcular [26], nematicidal insecticidal [17], anti-inflammatory and lipoxygenase [27]. Hence the authors have taken efforts to synthesis of some imines by perchloric acid catalyzed condensation of aryl amines with various substituted benzaldehydes. The synthesized Schiff's bases have been characterized by their analytical, physical constants and spectroscopic data. These data have been utilized for studying the biological activities of these imine derivatives which have been found out using Bauer-Kirby [28] method.

\section{EXPERIMENTAL}

All chemicals used in this work were purchased from Sigma-Aldrich Chemicals Private Limited. Melting points of all imines have been determined in open glass capillaries on Biom melting point apparatus (Universal Biochemicals Enzyme House, Madurai-3) and are uncorrected. Infrared spectra $\left(\mathrm{KBr}, 4000-400 \mathrm{~cm}^{-1}\right)$ have been recorded on Avatar-300 Fourier transform spectrophotometer (Thermo Nicolet, USA). The NMR spectra of all imines were recorded in Bruker AV400 spectrometer (Bruker AXS GmbH, Karlsruhe, Germany), operated $400 \mathrm{MHz}$ frequency for recording ${ }^{1} \mathrm{H}$ and $100 \mathrm{MHz}$ for ${ }^{13} \mathrm{C}$ NMR spectra in $\mathrm{CDCl}_{3}$ solvent using TMS as internal standard. Electron impact $(70 \mathrm{eV})$ and chemical ionization mode FAB + mass spectra have been recorded in Varian-Saturn 2200 GC-MS spectrometer (Varian 92 Medical Systems, Palo Alto, CA, USA).

\subsection{Synthesis of imines}

An appropriate equimolar quantity of aryl amines containing electron withdrawing and electron donating substituents $(1 \mathrm{mmol})$, substituted benzaldehydes ( $1 \mathrm{mmol})$, perchloric acid $(1 \mathrm{mmol})$ and ethanol $(20 \mathrm{~mL})$ were refluxed for about $4 \mathrm{~h}$ (Scheme 1). The completion of reaction 
was monitored by TLC. After completion of the reaction, the solid product was filtered using filter pump and dried. The crude product was recrystallized from ethanol.

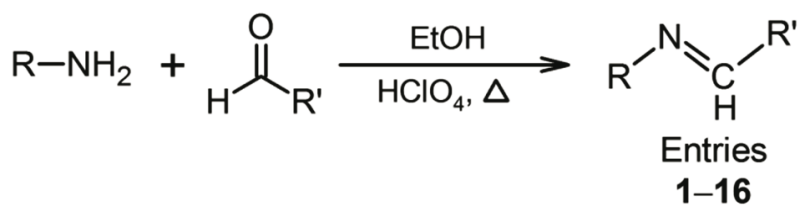

Scheme 1. Synthesis of imines by condensation of aldehydes and amines in presence of perchloric acid.

\subsection{Spectral data of entries $6-16$}

(E)-1-Benzyl-N-benzylidenepiperidin-4-amine (entry 6): Yield 81\%; m.p. 64-65 ${ }^{\circ} \mathrm{C}$; IR $\left(\mathrm{KBr}, \mathrm{cm}^{-1}\right): \mathrm{v}=1567.12(\mathrm{C}=\mathrm{N}) ;{ }^{1} \mathrm{H} \mathrm{NMR}\left(400 \mathrm{MHz}, \mathrm{CDCl}_{3}\right.$, ppm): $\delta=3.550$ (s, 2H, $\left.\mathrm{CH}_{2}\right), 8.327$ (s, $\left.1 \mathrm{H}, \mathrm{CH}\right), 1.707-3.278$ (m, 9H, alicyclic), 7.253-7.741 (m, 10H, Ar); ${ }^{13} \mathrm{C}$ NMR (100 MHz, $\left.\mathrm{CDCl}_{3}, \mathrm{ppm}\right): \delta=67.71\left(\mathrm{CH}_{2}\right)$, $159.16(\mathrm{CH}), 136.50\left(\mathrm{C}_{1}\right), 129.79\left(\mathrm{C}_{2}, \mathrm{C}_{6}\right), 130.50\left(\mathrm{C}_{4}\right), 128.58\left(\mathrm{C}_{3}, \mathrm{C}_{5}\right), 138.71$ $\left(\mathrm{C}_{1^{\prime}}\right), 128.21\left(\mathrm{C}_{2^{\prime}}, \mathrm{C}_{6^{\prime}}\right), 128.13\left(\mathrm{C}_{3^{\prime}}, \mathrm{C}_{5^{\prime}}\right), 126.25\left(\mathrm{C}_{4^{\prime}}\right), 52.51\left(\mathrm{C}_{2^{\prime \prime}}, \mathrm{C}_{6^{\prime \prime}}\right), 33.53$ $\left(\mathrm{C}_{3^{\prime \prime}}, \mathrm{C}_{5^{\prime \prime}}\right), 63.21\left(\mathrm{C}_{4^{\prime \prime}}\right)$; MF: $\mathrm{C}_{19} \mathrm{H}_{22} \mathrm{~N}_{2} ; \mathrm{MW}: 278$.

(E)-1-Benzyl-N-(3-bromobenzylidene)piperidin-4-amine (entry 7): Yield 80\%; m.p. 59-60 ${ }^{\circ} \mathrm{C}$; $\mathrm{IR}\left(\mathrm{KBr}, \mathrm{cm}^{-1}\right): \mathrm{v}=1565.44(\mathrm{C}=\mathrm{N}) ;{ }^{1} \mathrm{H} \mathrm{NMR}\left(400 \mathrm{MHz}, \mathrm{CDCl}_{3}\right.$, ppm): $\delta=3.510$ (s, 2H, $\mathrm{CH}_{2}$ ), 8.172 (s, 1H, CH), 7.198-7.888 (m, 9H, Ar), 1.6623.198 (m, 9H, alicyclic); ${ }^{13} \mathrm{C}$ NMR (100 MHz, $\left.\mathrm{CDCl}_{3}, \mathrm{ppm}\right): \delta=67.57\left(\mathrm{CH}_{2}\right)$, $157.54(\mathrm{CH}), 138.51\left(\mathrm{C}_{1}\right), 130.16\left(\mathrm{C}_{2}\right), 122.90\left(\mathrm{C}_{3}\right), 130.64\left(\mathrm{C}_{4}\right), 129.17\left(\mathrm{C}_{5}\right)$, $127.08\left(\mathrm{C}_{6}\right), 133.34\left(\mathrm{C}_{1^{\prime}}\right), 128.28\left(\mathrm{C}_{2^{\prime}}, \mathrm{C}_{6^{\prime}}\right), 127.05\left(\mathrm{C}_{3^{\prime}}, \mathrm{C}_{5^{\prime}}\right), 52.01\left(\mathrm{C}_{2^{\prime \prime}}, \mathrm{C}_{6^{\prime \prime}}\right)$, $33.46\left(\mathrm{C}_{3^{\prime \prime}}, \mathrm{C}_{5^{\prime \prime}}\right), 63.20\left(\mathrm{C}_{4^{\prime \prime}}\right)$; MF: $\mathrm{C}_{19} \mathrm{H}_{21} \mathrm{~N}_{2} \mathrm{Br}$; MW: 357.

(E)-1-Benzyl-N-(3-chlorobenzylidene)piperidin-4-amine (entry 8):Yield 83\%; m.p. $53-54^{\circ} \mathrm{C}$; IR $\left(\mathrm{KBr}, \mathrm{cm}^{-1}\right): v=1595.71(\mathrm{C}=\mathrm{N}) ;{ }^{1} \mathrm{H}$ NMR $\left(400 \mathrm{MHz}, \mathrm{CDCl}_{3}\right.$, ppm): $\delta=3.538$ (s, 2H, CH${ }_{2}$ ), 8.236 (s, 1H, CH), 1.689-3.267 (m, 9H, alicyclic), 7.225-7.750 (m, 9H, Ar); ${ }^{13} \mathrm{C}$ NMR (100 MHz, $\left.\mathrm{CDCl}_{3}, \mathrm{ppm}\right): \delta=67.66\left(\mathrm{CH}_{2}\right)$, $157.69(\mathrm{CH}), 138.62\left(\mathrm{C}_{1}\right), 127.05\left(\mathrm{C}_{2}\right), 134.78\left(\mathrm{C}_{3}\right), 130.48\left(\mathrm{C}_{4}\right), 129.87\left(\mathrm{C}_{5}\right)$, $127.77\left(\mathrm{C}_{6}\right), 138.29\left(\mathrm{C}_{1^{\prime}}\right), 128.62\left(\mathrm{C}_{2^{\prime}}, \mathrm{C}_{6^{\prime}}\right), 128.29\left(\mathrm{C}_{3^{\prime}}, \mathrm{C}_{5^{\prime}}\right), 126.59\left(\mathrm{C}_{6^{\prime}}\right), 52.05\left(\mathrm{C}_{2^{\prime \prime}}\right.$, $\left.\mathrm{C}_{6^{\prime \prime}}\right), 33.49\left(\mathrm{C}_{3^{\prime \prime}}, \mathrm{C}_{5^{\prime \prime}}\right), 63.23\left(\mathrm{C}_{4^{\prime \prime}}\right) ; \mathrm{MF}: \mathrm{C}_{19} \mathrm{H}_{21} \mathrm{~N}_{2} \mathrm{Cl}$; MW: 312.

(E)-1-Benzyl-N-(4-chlorobenzylidene)piperidin-4-amine (entry 9):Yield 82\%; m.p. $102-103^{\circ} \mathrm{C}$; IR $\left(\mathrm{KBr}, \mathrm{cm}^{-1}\right): v=1592.24(\mathrm{C}=\mathrm{N}) ;{ }^{1} \mathrm{H} \mathrm{NMR}\left(400 \mathrm{MHz}, \mathrm{CDCl}_{3}\right.$, ppm): $\delta=3.543$ (s, $2 \mathrm{H}, \mathrm{CH}_{2}$ ), 8.271 (s, 1H, CH), 1.690-3.234 (m, 9H, alicyclic), 7.251-7.668 (m, 9H, Ar); ${ }^{13} \mathrm{C}$ NMR (100 MHz, $\left.\mathrm{CDCl}_{3}, \mathrm{ppm}\right): \delta=67.70\left(\mathrm{CH}_{2}\right)$, $157.80(\mathrm{CH}), 134.97\left(\mathrm{C}_{1}\right), 129.35\left(\mathrm{C}_{2}, \mathrm{C}_{6}\right), 128.84\left(\mathrm{C}_{3}, \mathrm{C}_{5}\right), 136.39\left(\mathrm{C}_{4}\right), 138.66$ 
$\left(\mathrm{C}_{1^{\prime}}\right), 128.39\left(\mathrm{C}_{2^{\prime}}, \mathrm{C}_{6^{\prime}}\right), 128.24\left(\mathrm{C}_{3^{\prime}}, \mathrm{C}_{5^{\prime}}\right), 126.99\left(\mathrm{C}_{4^{\prime}}\right), 52.10\left(\mathrm{C}_{2^{\prime \prime}}, \mathrm{C}_{6^{\prime \prime}}\right), 33.50$ $\left(\mathrm{C}_{3^{\prime \prime}}, \mathrm{C}_{5^{\prime \prime}}\right), 63.23\left(\mathrm{C}_{4^{\prime \prime}}\right)$; $\mathrm{MF}: \mathrm{C}_{19} \mathrm{H}_{21} \mathrm{~N}_{2} \mathrm{Cl}$; MW: 312 .

(E)-1-Benzyl-N-(4-(dimethylamino)benzylidine)piperidin-4-amine (entry 10): Yield 80\%; m.p. $57-58^{\circ} \mathrm{C}$; IR $\left(\mathrm{KBr}, \mathrm{cm}^{-1}\right): \mathrm{v}=1560.07(\mathrm{C}=\mathrm{N}) ;{ }^{1} \mathrm{H}$ NMR (400 $\left.\mathrm{MHz}, \mathrm{CDCl}_{3}, \mathrm{ppm}\right): \delta=3.540\left(\mathrm{~s}, 2 \mathrm{H}, \mathrm{CH}_{2}\right), 8.190(\mathrm{~s}, 1 \mathrm{H}, \mathrm{CH}), 1.685-3.185(\mathrm{~m}$, 9H, alicyclic), 6.667-7.749 (m, 9H, Ar), $3.325\left(\mathrm{~s}, 6 \mathrm{H}, 2 \mathrm{CH}_{3}\right) ;{ }^{13} \mathrm{C} \mathrm{NMR}(100$ $\left.\mathrm{MHz}, \mathrm{CDCl}_{3}, \mathrm{ppm}\right): \delta=67.64\left(\mathrm{CH}_{2}\right), 159.14(\mathrm{CH}), 125.19\left(\mathrm{C}_{1}\right), 124.70\left(\mathrm{C}_{2}, \mathrm{C}_{6}\right)$, $111.66\left(\mathrm{C}_{3}, \mathrm{C}_{5}\right), 152.00\left(\mathrm{C}_{4}\right), 138.72\left(\mathrm{C}_{1^{\prime}}\right), 129.19\left(\mathrm{C}_{2^{\prime}}, \mathrm{C}_{6^{\prime}}\right), 128.20\left(\mathrm{C}_{3^{\prime}}, \mathrm{C}_{5^{\prime}}\right)$, $126.99\left(\mathrm{C}_{4^{\prime}}\right), 52.36\left(\mathrm{C}_{2^{\prime \prime}}, \mathrm{C}_{6^{\prime \prime}}\right), 33.73\left(\mathrm{C}_{3^{\prime \prime}}, \mathrm{C}_{5^{\prime \prime}}\right), 63.17\left(\mathrm{C}_{4^{\prime \prime}}\right), 41.04\left(\mathrm{CH}_{3}\right) ; \mathrm{MF}$ : $\mathrm{C}_{21} \mathrm{H}_{27} \mathrm{~N}_{3}$; MW: 321 .

(E)-1-Benzyl-N-(4-fluorobenzylidine)piperidin-4-amine (entry 11):Yield 80\%; m.p. 92-93 ${ }^{\circ} \mathrm{C}$; IR $\left(\mathrm{KBr}, \mathrm{cm}^{-1}\right): \mathrm{v}=1597.18(\mathrm{C}=\mathrm{N}) ;{ }^{1} \mathrm{H} \mathrm{NMR}\left(400 \mathrm{MHz}, \mathrm{CDCl}_{3}\right.$, ppm): $\delta=3.547$ (s, 2H, $\mathrm{CH}_{2}$ ), 8.282 (s, 1H, CH), 1.729-3.239 (m, 9H, alicyclic), 7.057-7.736 (m, 9H, Ar); ${ }^{13} \mathrm{C}$ NMR (100 MHz, $\left.\mathrm{CDCl}_{3}, \mathrm{ppm}\right): \delta=67.56\left(\mathrm{CH}_{2}\right)$, $157.68(\mathrm{CH}), 132.81\left(\mathrm{C}_{1}\right), 130.03\left(\mathrm{C}_{2}, \mathrm{C}_{6}\right), 115.74\left(\mathrm{C}_{3}, \mathrm{C}_{5}\right), 165.45\left(\mathrm{C}_{4}\right), 138.63$ $\left(\mathrm{C}_{1^{\prime}}\right), 129.95\left(\mathrm{C}_{2^{\prime}}, \mathrm{C}_{6^{\prime}}\right), 128.23\left(\mathrm{C}_{3^{\prime}}, \mathrm{C}_{5^{\prime}}\right), 126.98\left(\mathrm{C}_{4^{\prime}}\right), 52.10\left(\mathrm{C}_{2^{\prime \prime}}, \mathrm{C}_{6^{\prime \prime}}\right), 33.52$ $\left(\mathrm{C}_{3^{\prime \prime}}, \mathrm{C}_{5^{\prime \prime}}\right), 63.20\left(\mathrm{C}_{4^{\prime \prime}}\right)$; MF: $\mathrm{C}_{19} \mathrm{H}_{21} \mathrm{~N}_{2} \mathrm{~F} ; \mathrm{MW}: 296$.

(E)-1-Benzyl-N-(3-methoxybenzylidine)piperidin-4-amine (entry 12): Yield 86\%; m.p. 63-64 ${ }^{\circ} \mathrm{C}$; IR $\left(\mathrm{KBr}, \mathrm{cm}^{-1}\right): \mathrm{v}=1593.42(\mathrm{C}=\mathrm{N}) ;{ }^{1} \mathrm{H}$ NMR $(400 \mathrm{MHz}$, $\left.\mathrm{CDCl}_{3}, \mathrm{ppm}\right): \delta=3.552\left(\mathrm{~s}, 2 \mathrm{H}, \mathrm{CH}_{2}\right), 8.294(\mathrm{~s}, 1 \mathrm{H}, \mathrm{CH}), 1.708-3.280(\mathrm{~m}, 9 \mathrm{H}$, alicyclic), 7.239-7.363 (m, 9H, Ar), 3.851 (s, 3H, $\left.\mathrm{OCH}_{3}\right) ;{ }^{13} \mathrm{C}$ NMR (100 MHz, $\left.\mathrm{CDCl}_{3}, \mathrm{ppm}\right): \delta=67.63\left(\mathrm{CH}_{2}\right), 159.88(\mathrm{CH}), 138.66\left(\mathrm{C}_{1}\right), 111.81\left(\mathrm{C}_{2}\right), 159.01\left(\mathrm{C}_{3}\right)$, $117.09\left(\mathrm{C}_{4}\right), 129.55\left(\mathrm{C}_{5}\right), 121.33\left(\mathrm{C}_{6}\right), 55.41(3-\mathrm{OMe}), 137.98\left(\mathrm{C}_{1^{\prime}}\right), 129.14\left(\mathrm{C}_{2^{\prime}}\right.$, $\left.\mathrm{C}_{6^{\prime}}\right), 128.21\left(\mathrm{C}_{3^{\prime}}, \mathrm{C}_{5^{\prime}}\right), 126.96\left(\mathrm{C}_{4^{\prime}}\right), 52.14\left(\mathrm{C}_{2^{\prime \prime}}, \mathrm{C}_{6^{\prime \prime}}\right), 33.49\left(\mathrm{C}_{3^{\prime \prime}}, \mathrm{C}_{5^{\prime \prime}}\right), 63.19\left(\mathrm{C}_{4^{\prime \prime}}\right)$; MF: $\mathrm{C}_{20} \mathrm{H}_{24} \mathrm{~N}_{2} \mathrm{O} ; \mathrm{MW}: 308$.

(E)-1-Benzyl-N-(4-methoxybenzylidine)piperidin-4-amine (entry 13): Yield 82\%; m.p. 49-50 ${ }^{\circ} \mathrm{C}$; IR $\left(\mathrm{KBr}, \mathrm{cm}^{-1}\right): \mathrm{v}=1575.79(\mathrm{C}=\mathrm{N}) ;{ }^{1} \mathrm{H}$ NMR (400 MHz, $\left.\mathrm{CDCl}_{3}, \mathrm{ppm}\right): \delta=3.534\left(\mathrm{~s}, 2 \mathrm{H}, \mathrm{CH}_{2}\right), 8.235(\mathrm{~s}, 1 \mathrm{H}, \mathrm{CH}), 3.795\left(\mathrm{~s}, 3 \mathrm{H}, \mathrm{OCH}_{3}\right)$, 1.712-3.216 (m, 9H, alicyclic), 6.882-7.672 (m, 9H, Ar); ${ }^{13} \mathrm{C}$ NMR (100 MHz, $\left.\mathrm{CDCl}_{3}, \mathrm{ppm}\right): \delta=67.67\left(\mathrm{CH}_{2}\right), 161.52(\mathrm{CH}), 128.95\left(\mathrm{C}_{1}\right), 130.54\left(\mathrm{C}_{2}, \mathrm{C}_{6}\right)$, $114.38\left(\mathrm{C}_{3}, \mathrm{C}_{5}\right), 164.65\left(\mathrm{C}_{4}\right), 55.38\left(4-\mathrm{OCH}_{3}\right), 138.72\left(\mathrm{C}_{1^{\prime}}\right), 128.61\left(\mathrm{C}_{2^{\prime}}, \mathrm{C}_{6^{\prime}}\right)$, $128.18\left(\mathrm{C}_{3^{\prime}}, \mathrm{C}_{5^{\prime}}\right), 126.66\left(\mathrm{C}_{4^{\prime}}\right), 52.50\left(\mathrm{C}_{2^{\prime \prime}}, \mathrm{C}_{6^{\prime \prime}}\right), 33.84\left(\mathrm{C}_{3^{\prime \prime}}, \mathrm{C}_{5^{\prime \prime}}\right), 63.24\left(\mathrm{C}_{4^{\prime \prime}}\right) ; \mathrm{MF}$ : $\mathrm{C}_{20} \mathrm{H}_{24} \mathrm{~N}_{2} \mathrm{O} ; \mathrm{MW}: 308$.

(E)-1-Benzyl-N-(4-methylbenzylidine)piperidin-4-amine (entry 14):Yield 87\%; m.p. $51-52^{\circ} \mathrm{C}$; IR $\left(\mathrm{KBr}, \mathrm{cm}^{-1}\right): \mathrm{v}=1573.95(\mathrm{C}=\mathrm{N}) ;{ }^{1} \mathrm{H} \mathrm{NMR}\left(400 \mathrm{MHz}, \mathrm{CDCl}_{3}\right.$, ppm): $\delta=3.547$ (s, 1H, $\mathrm{CH}_{2}$ ), 8.285 (s, 1H, CH), 1.698-2.249 (m, 9H, alicyclic), 7.187-7.625 (m, 9H, Ar), $2.369\left(\mathrm{~s}, 3 \mathrm{H}, \mathrm{CH}_{3}\right) ;{ }^{13} \mathrm{C} \mathrm{NMR} \mathrm{(100} \mathrm{MHz}, \mathrm{CDCl}_{3}$, ppm): $\delta=67.70\left(\mathrm{CH}_{2}\right), 159.09(\mathrm{CH}), 133.90\left(\mathrm{C}_{1}\right), 129.29\left(\mathrm{C}_{2}, \mathrm{C}_{6}\right), 129.14\left(\mathrm{C}_{3}, \mathrm{C}_{5}\right)$, $140.71\left(\mathrm{C}_{4}\right), 138.70\left(\mathrm{C}_{1^{\prime}}\right), 128.21\left(\mathrm{C}_{2^{\prime}}, \mathrm{C}_{6^{\prime}}\right), 128.11\left(\mathrm{C}_{3^{\prime}}, \mathrm{C}_{5^{\prime}}\right), 126.95\left(\mathrm{C}_{4^{\prime}}\right), 52.20\left(\mathrm{C}_{2^{\prime \prime}}\right.$, $\left.\mathrm{C}_{6^{\prime \prime}}\right), 33.56\left(\mathrm{C}_{3^{\prime \prime}}, \mathrm{C}_{5^{\prime \prime}}\right), 63.19\left(\mathrm{C}_{4^{\prime \prime}}\right), 21.53\left(4-\mathrm{CH}_{3}\right) ; \mathrm{MF}: \mathrm{C}_{20} \mathrm{H}_{24} \mathrm{~N}_{2} ; \mathrm{MW}: 292$. 
(E)-1-Benzyl-N-(3-nitrobenzylidine)piperidin-4-amine (entry 15): Yield 82\%; m.p. $60-61^{\circ} \mathrm{C}$; IR $\left(\mathrm{KBr}, \mathrm{cm}^{-1}\right): \mathrm{v}=1526.95(\mathrm{C}=\mathrm{N}) ;{ }^{1} \mathrm{H} \mathrm{NMR}\left(400 \mathrm{MHz}, \mathrm{CDCl}_{3}\right.$, ppm): $\delta=3.580$ (s, 1H, $\left.\mathrm{CH}_{2}\right), 8.556$ (s, 1H, CH), 1.742-3.356 (m, 9H, alicyclic), 7.261-8.390 (m, 9H, Ar); ${ }^{13} \mathrm{C}$ NMR (100 MHz, $\left.\mathrm{CDCl}_{3}, \mathrm{ppm}\right): \delta=67.45\left(\mathrm{CH}_{2}\right)$, $156.51(\mathrm{CH}), 138.35\left(\mathrm{C}_{1}\right), 122.97\left(\mathrm{C}_{2}\right), 148.60\left(\mathrm{C}_{3}\right), 124.92\left(\mathrm{C}_{4}\right), 129.57\left(\mathrm{C}_{5}\right), 133.61$ $\left(\mathrm{C}_{6}\right), 138.18\left(\mathrm{C}_{1^{\prime}}\right), 129.20\left(\mathrm{C}_{2^{\prime}}, \mathrm{C}_{6^{\prime}}\right), 128.26\left(\mathrm{C}_{3^{\prime}}, \mathrm{C}_{5^{\prime}}\right), 127.08\left(\mathrm{C}_{4^{\prime}}\right), 51.83\left(\mathrm{C}_{2^{\prime \prime}}, \mathrm{C}_{6^{\prime \prime}}\right), 33.31$ $\left(\mathrm{C}_{3^{\prime \prime}}, \mathrm{C}_{5^{\prime \prime}}\right), 63.13\left(\mathrm{C}_{4^{\prime \prime}}\right)$; MF: $\mathrm{C}_{19} \mathrm{H}_{21} \mathrm{~N}_{3} \mathrm{O}_{2} ; \mathrm{MW}: 323$.

(E)-1-Benzyl-N-(4-nitrobenzylidine)piperidin-4-amine (entry 16): Yield 85\%; m.p. 82-83 ${ }^{\circ}$; IR $\left(\mathrm{KBr}, \mathrm{cm}^{-1}\right): \mathrm{v}=1598.42(\mathrm{C}=\mathrm{N}) ;{ }^{1} \mathrm{H} \mathrm{NMR}\left(400 \mathrm{MHz} \mathrm{CDCl}_{3}\right.$, ppm): $\delta=3.559$ (s, 2H, $\mathrm{CH}_{2}$ ), 8.400 (s, 1H, CH), 1.723-3.362 (m, 9H, alicyclic), 7.260-8.265 (m, 9H, Ar); ${ }^{13} \mathrm{C}$ NMR (100 MHz, $\left.\mathrm{CDCl}_{3}, \mathrm{ppm}\right): \delta=67.89\left(\mathrm{CH}_{2}\right)$, $156.81(\mathrm{CH}), 141.99\left(\mathrm{C}_{1}\right), 129.14\left(\mathrm{C}_{2}, \mathrm{C}_{6}\right), 123.86\left(\mathrm{C}_{3}, \mathrm{C}_{5}\right), 148.93\left(\mathrm{C}_{4}\right), 138.55$ $\left(\mathrm{C}_{1^{\prime}}\right), 128.82\left(\mathrm{C}_{2^{\prime}}, \mathrm{C}_{6^{\prime}}\right), 128.25\left(\mathrm{C}_{3^{\prime}}, \mathrm{C}_{5^{\prime}}\right), 127.03\left(\mathrm{C}_{4^{\prime}}\right), 51.92\left(\mathrm{C}_{2^{\prime \prime}}, \mathrm{C}_{6^{\prime \prime}}\right), 33.37$ $\left(\mathrm{C}_{3^{\prime \prime}}, \mathrm{C}_{5^{\prime \prime}}\right), 63.21\left(\mathrm{C}_{4^{\prime \prime}}\right)$; MF: $\mathrm{C}_{19} \mathrm{H}_{21} \mathrm{~N}_{3} \mathrm{O}_{2}, \mathrm{MW}: 323$.

\subsection{Antibacterial sensitivity assay}

In this present study, antibacterial activities of synthesized imines (entries 6-16) against their respective microbes such as bacterial strains were studied. The antibacterial assays have been performed by using Bauer-Kirby [28] disc diffusion technique. In each petri plate about $0.5 \mathrm{~cm}^{3}$ of the test bacterial sample has been spread uniformly over the solidified Mueller Hinton agar using sterile glass spreader. Then the discs with $5 \mathrm{~mm}$ diameter made from Whatman No. 1 filter paper, impregnated with the solution of the compound have been placed on the medium using sterile forceps. The plates have been incubated for $24 \mathrm{~h}$ at $37^{\circ} \mathrm{C}$ by keeping the plates upside down to prevent the collection of water droplets over the medium. After $24 \mathrm{~h}$, the plates have been visually examined and the diameters of the zone of inhibition were measured. Triplicate results have been recorded by repeating the same procedure.

\section{RESULTS AND DISCUSSION}

In organic chemistry research laboratory, works have been carried out to synthesize aryl imine derivatives by condensation of aryl amines and various benzaldehydes containing electron withdrawing as well as electron donating substituents. The condensation reaction is feasible with aryl amines and benzaldehydes in the presence of vigorous acidic catalyst like perchloric acid in ethanol except acidic salt or base or its salt in 
solution media under reflux condition in atmospheric temperature and pressure. Hence the authors have synthesized the imine derivatives by the condensation reaction between $1 \mathrm{mmol}$ of aryl amines, $1 \mathrm{mmol}$ substituteed benzaldehydes, $1 \mathrm{mmol}$ of perchloric acid and $20 \mathrm{~cm}^{3}$ of ethanol at room temperature (Scheme 1). During the course of this reaction the perchloric acid catalyzes coupling between aryl amines and aldehydes by elimination of water followed by loss of proton forms the imines. The yield of the imines in this reaction is more than $80 \%$. The proposed general mechanism of this reaction is given in Scheme 2.

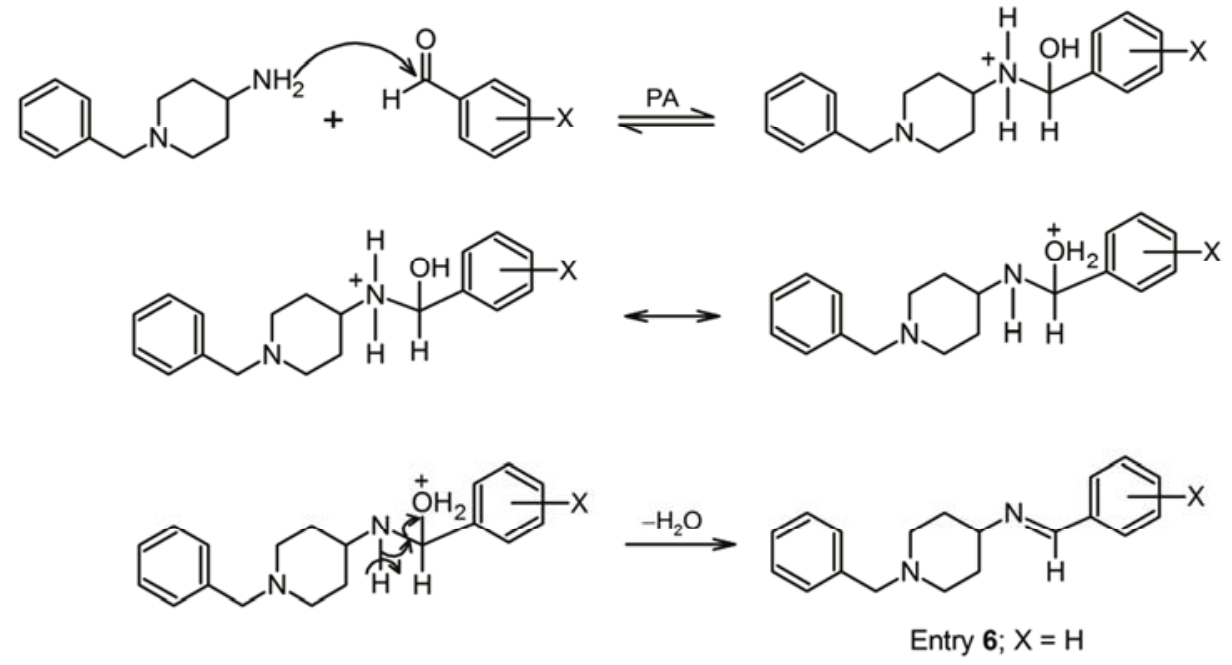

Scheme 2. Proposed mechanism of formation of imines by condensation of aldehydes and amines in presence of perchloric acid.

Further, we have also investigated this reaction with equimolar quantities of 4-amino-1-benzylpiperidine and benzaldehyde (entry 6). In this reaction the product yield is $85 \%$. The physical constants, yield and mass spectral data are presented in Table 1. IR and NMR spectroscopic data and mass fragment pattern of selective compounds are given in Table 2 and Schemes 3-6. 


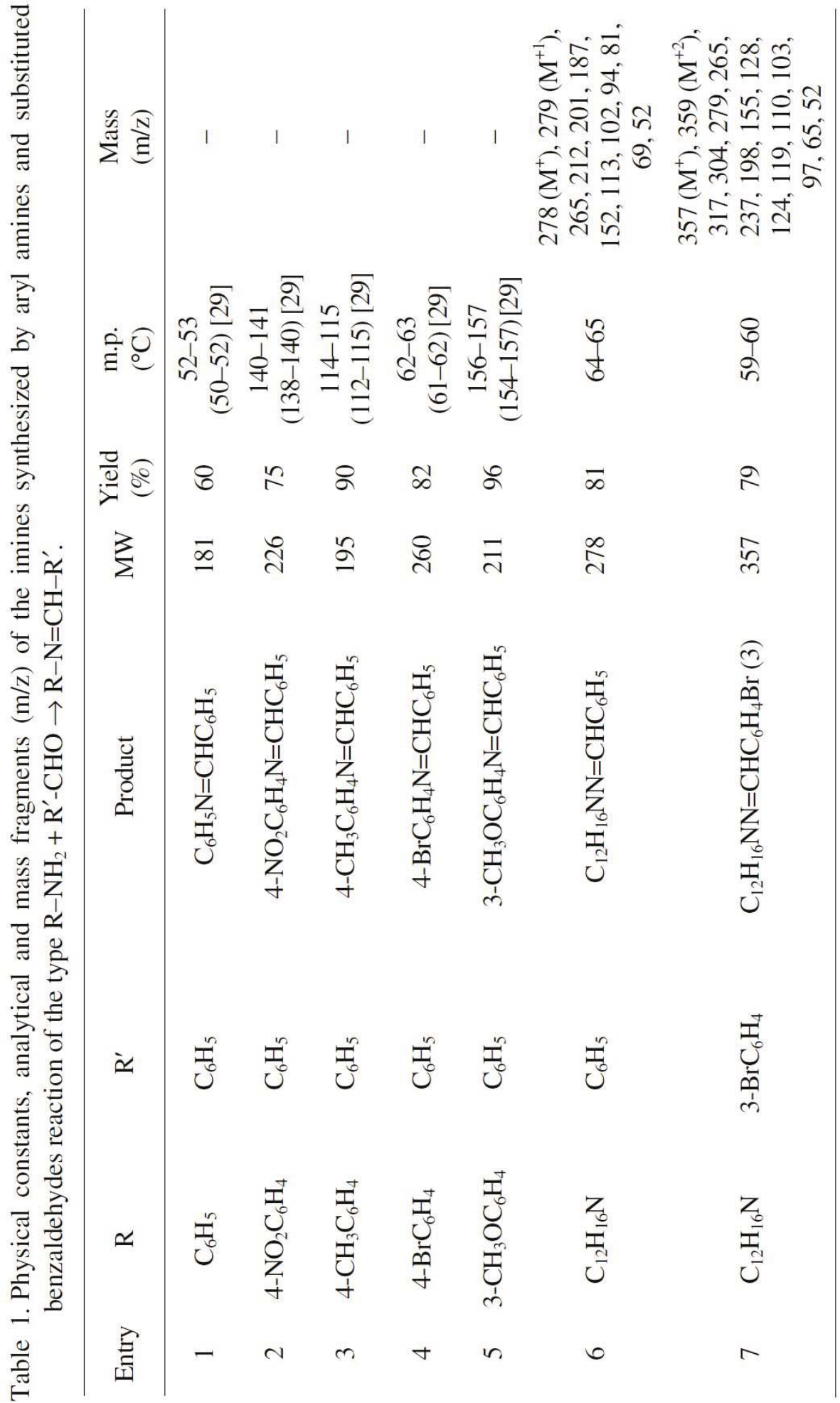




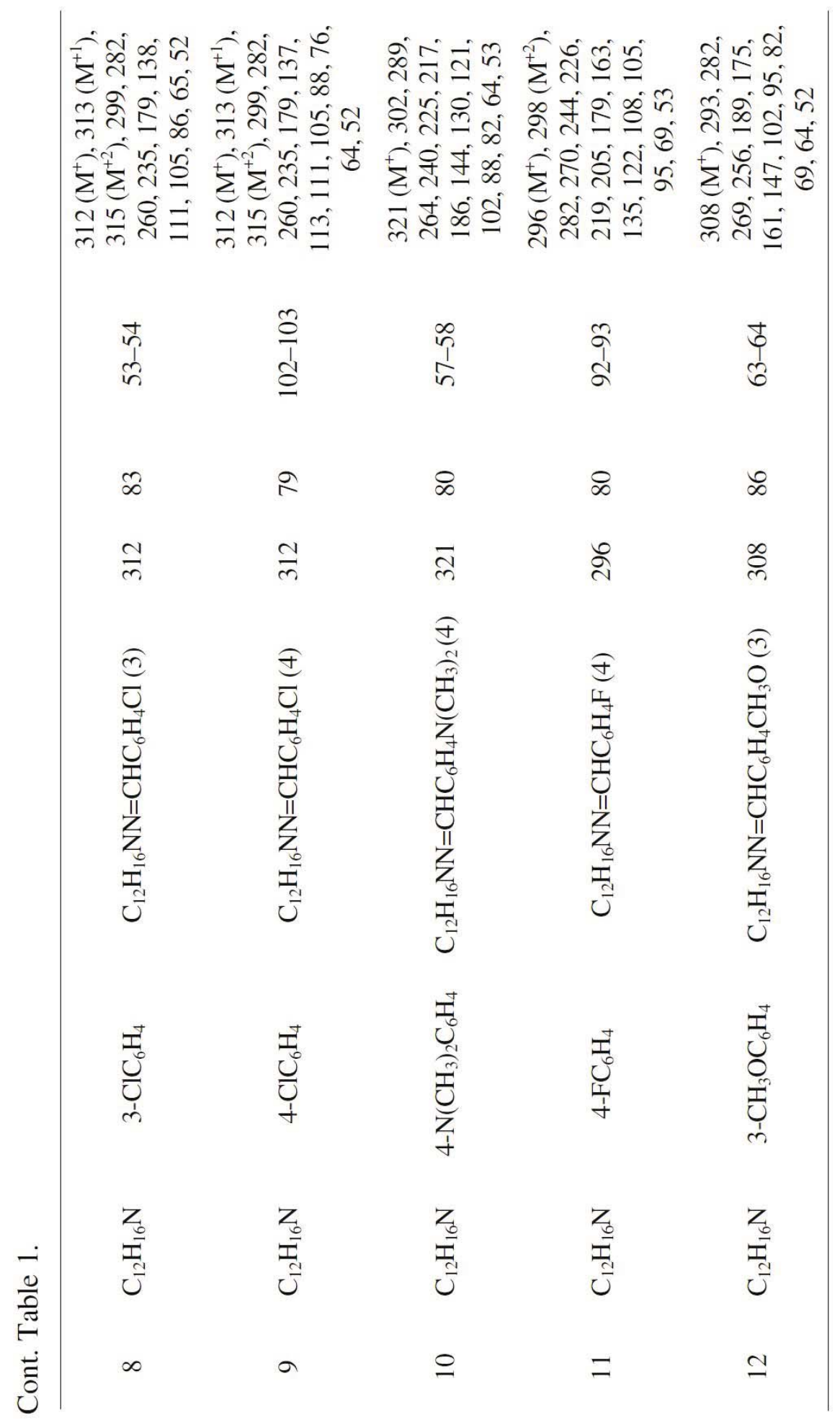




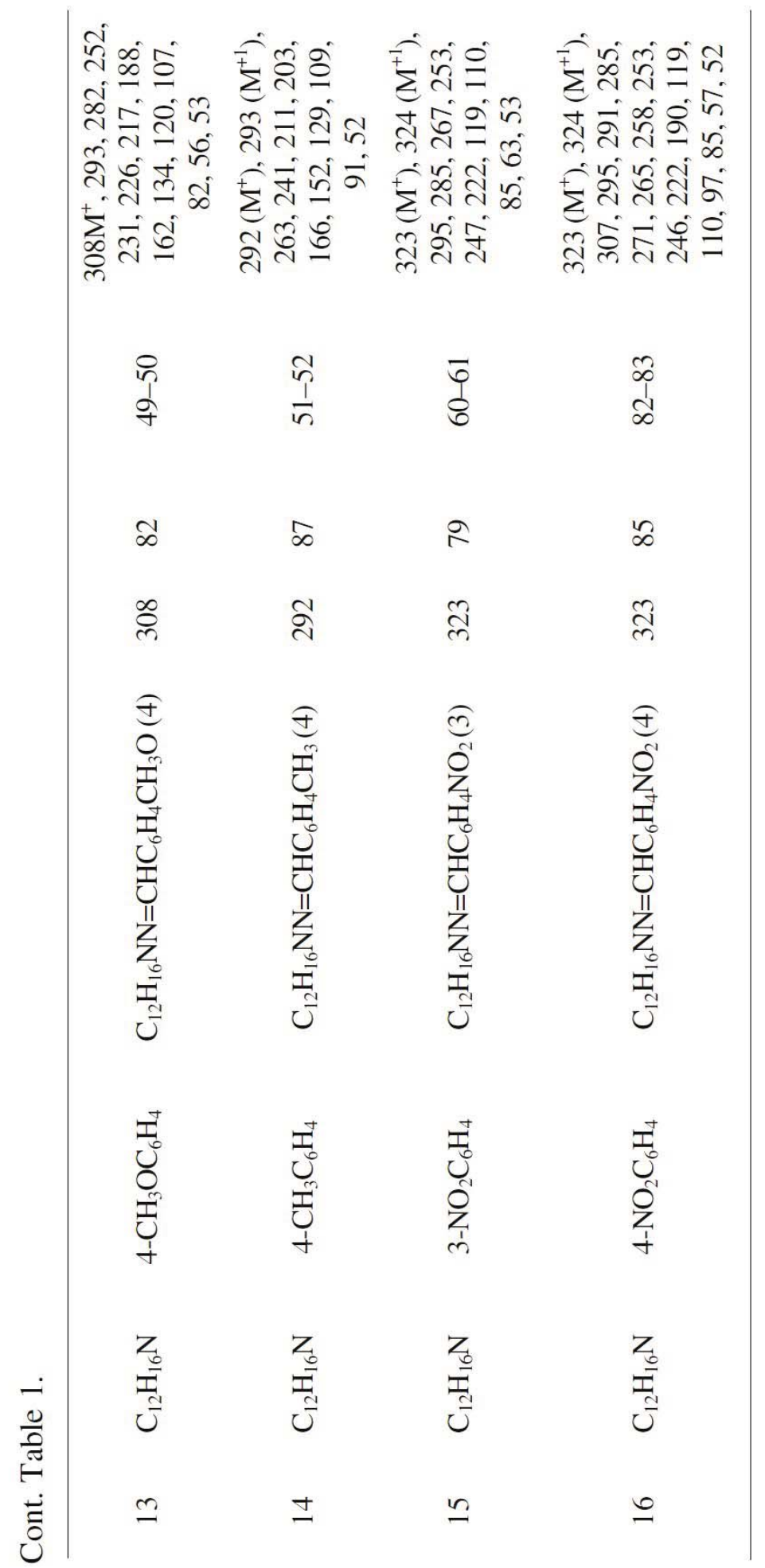




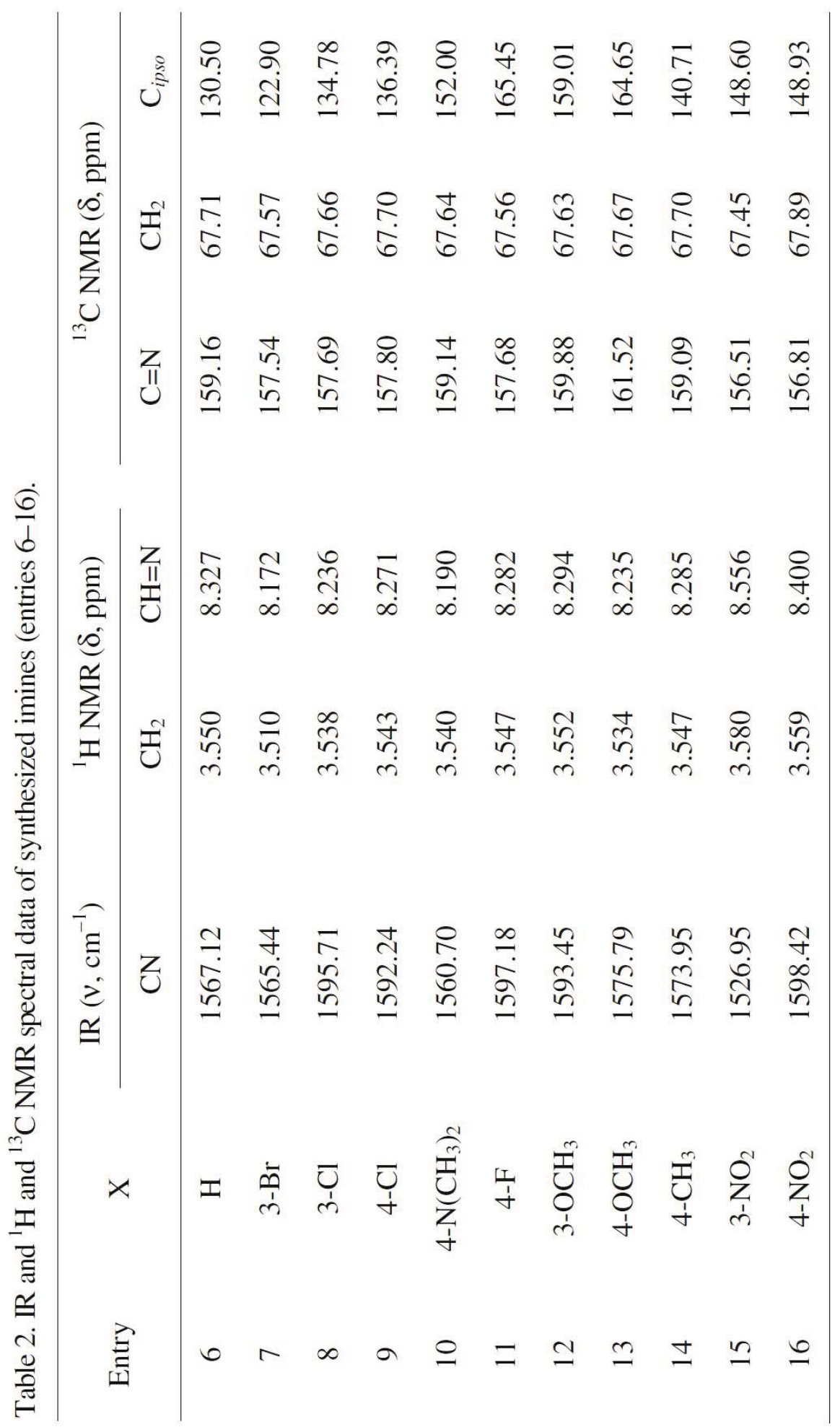




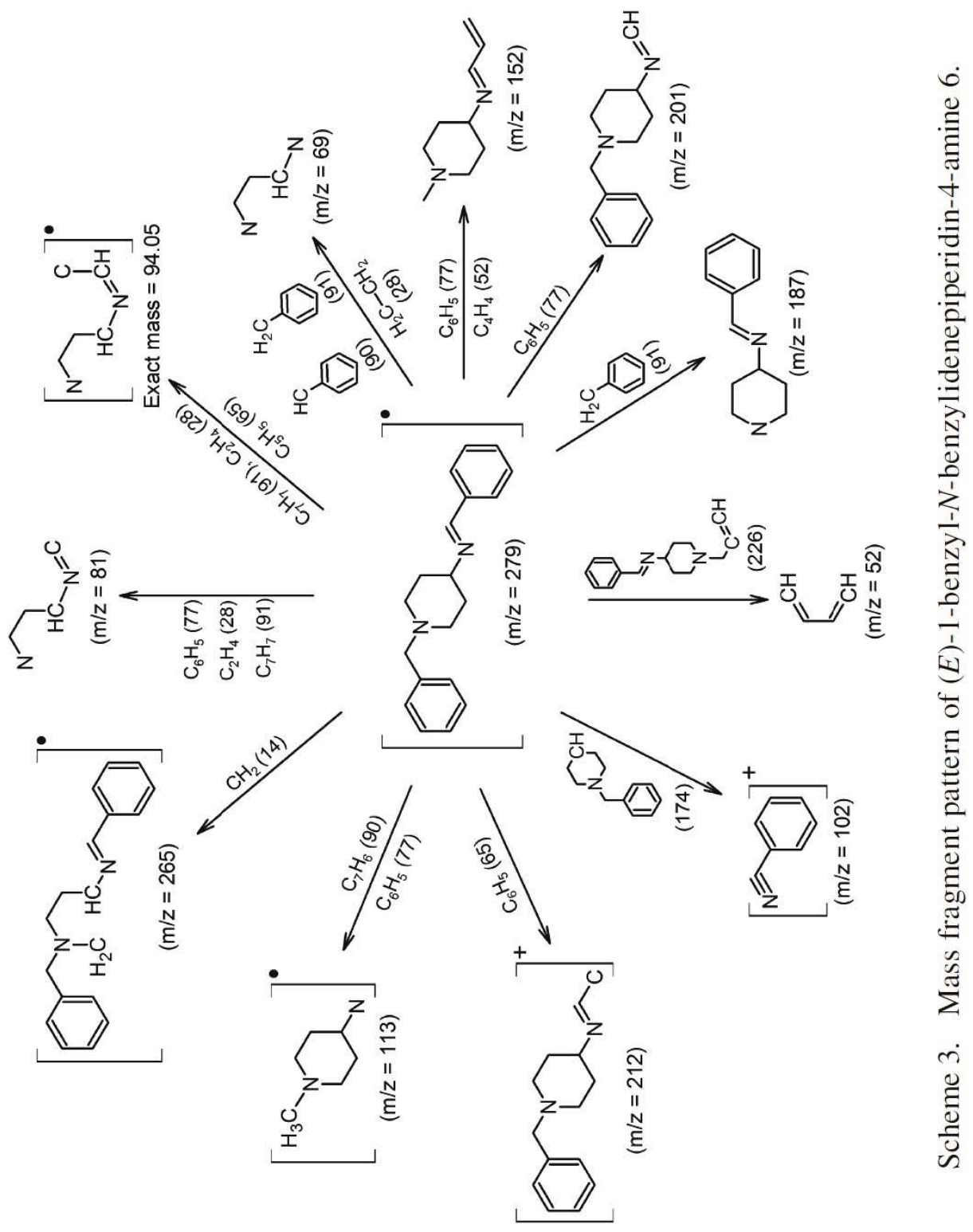




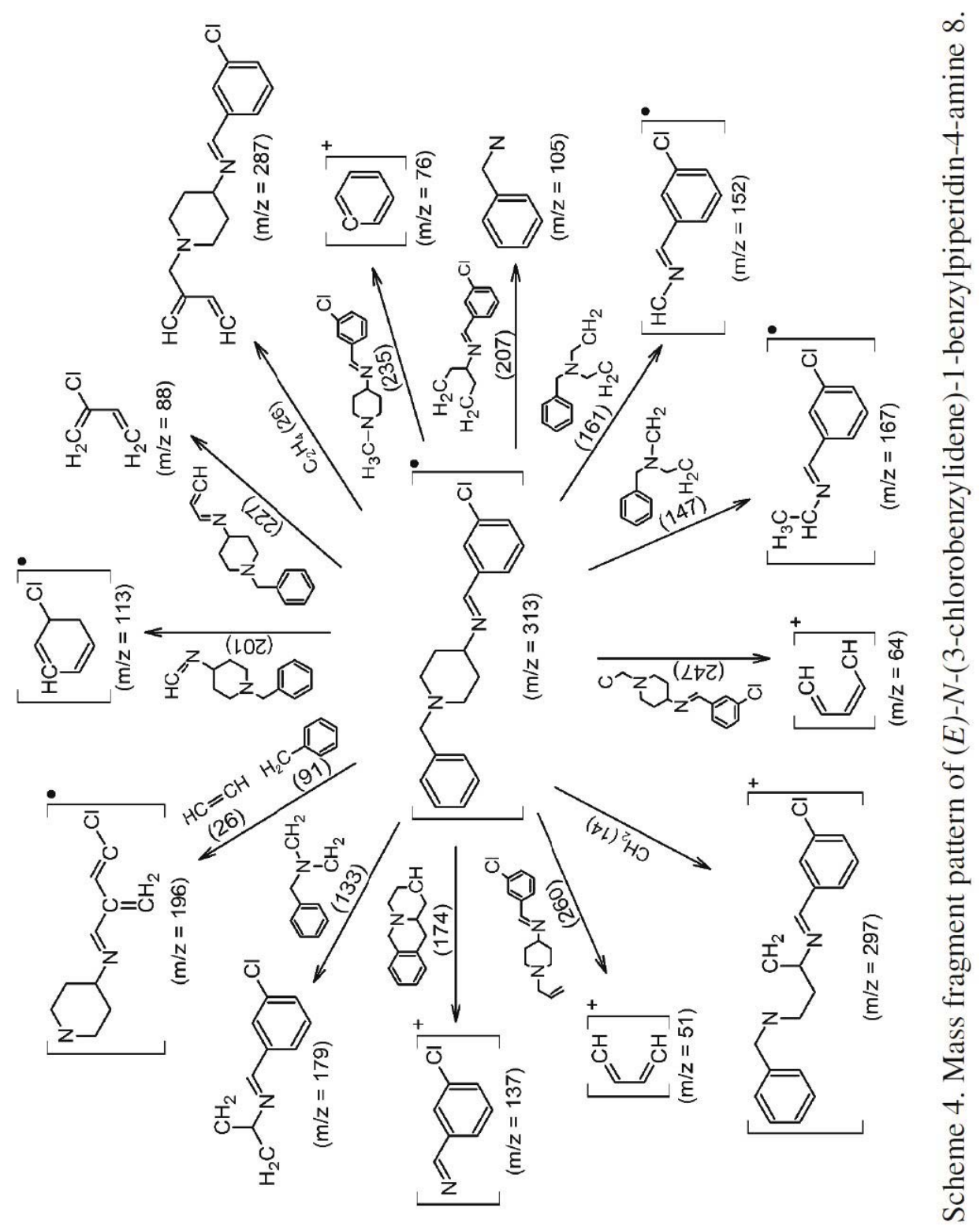




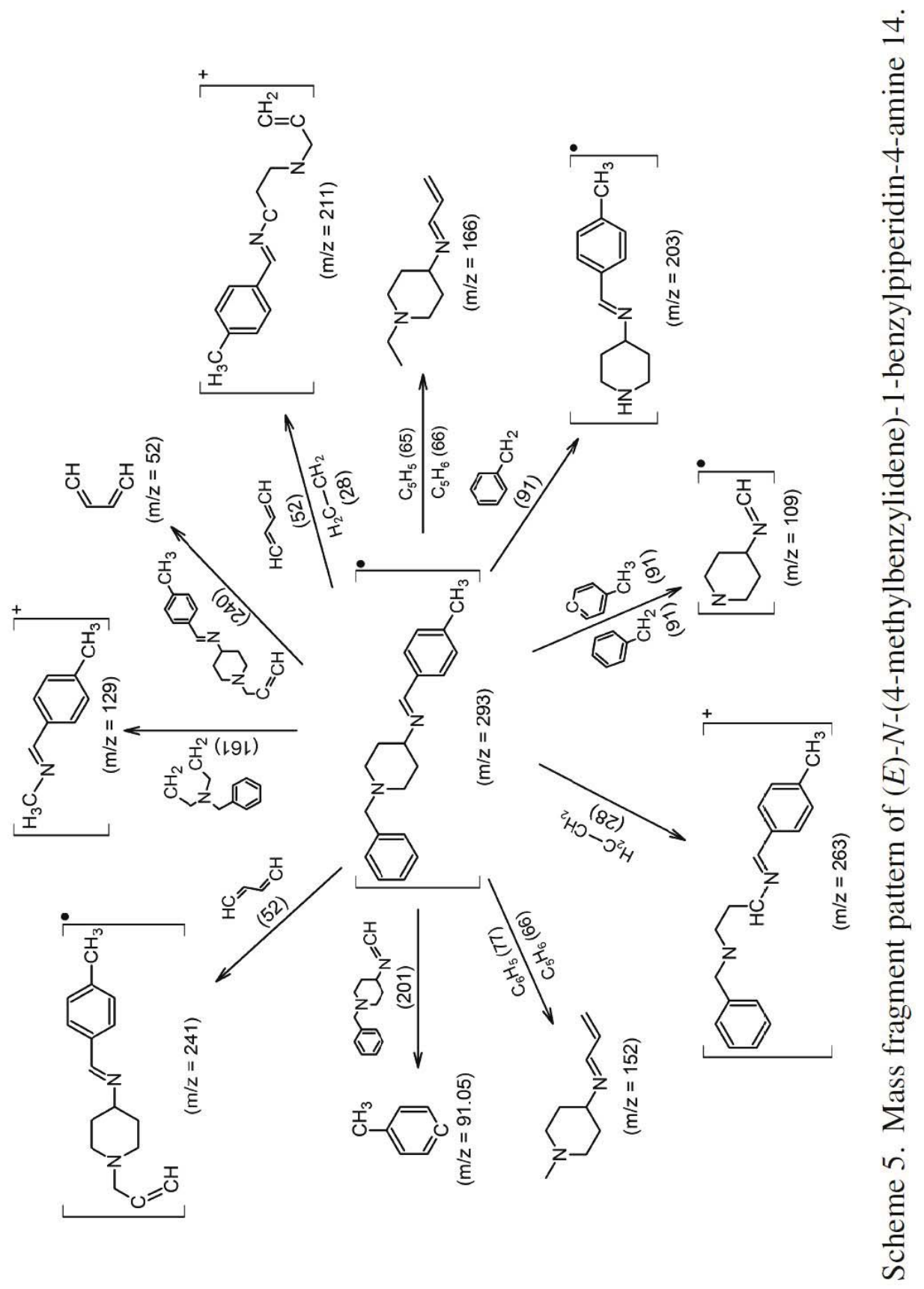




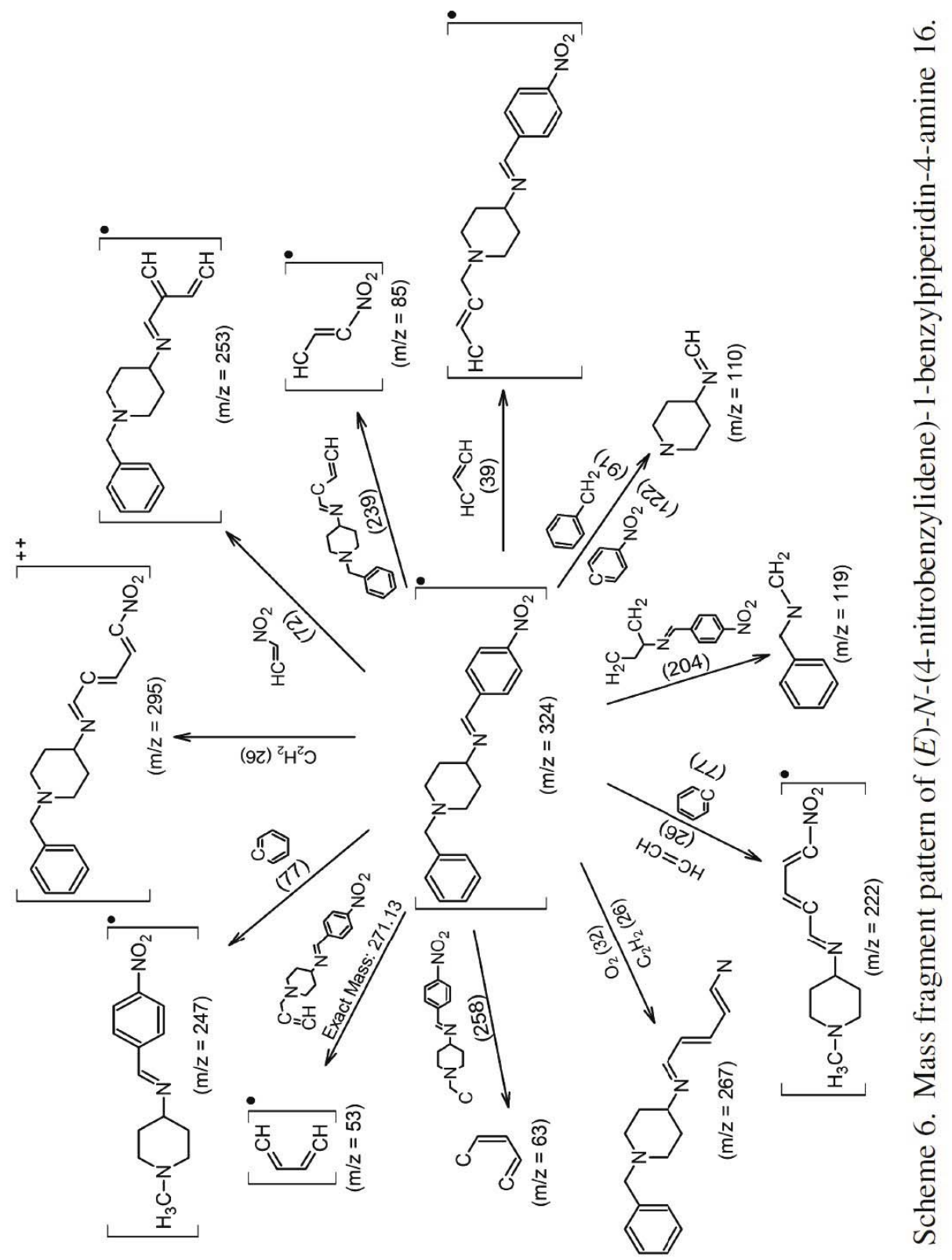


We have studied the effect of solvent for this condensation of amines and benzaldehydes by observing the yield of the products. The solvents like formic acid, acetic acid, dioxane, methanol, dichloromethane, dimethylformamide (DMF), tetrahydrofuran (THF) and toluene have been used for this reaction with 4-amino-1-benzylpiperidine and benzaldehyde. The percentage of products with various solvents is shown in Table 3 and the statistical diagram is shown in Fig. 1. Carrying out this condensation reaction with above solvents the observed yields are $73 \%$, $68 \%, 65 \%, 69 \%, 75 \%, 70 \%, 78 \%$ and $71 \%$, respectively. Here the authors have observed the good yield of imines in this synthetic method by condensation of amine and aldehydes in presence of perchloric acid at reflux condition.

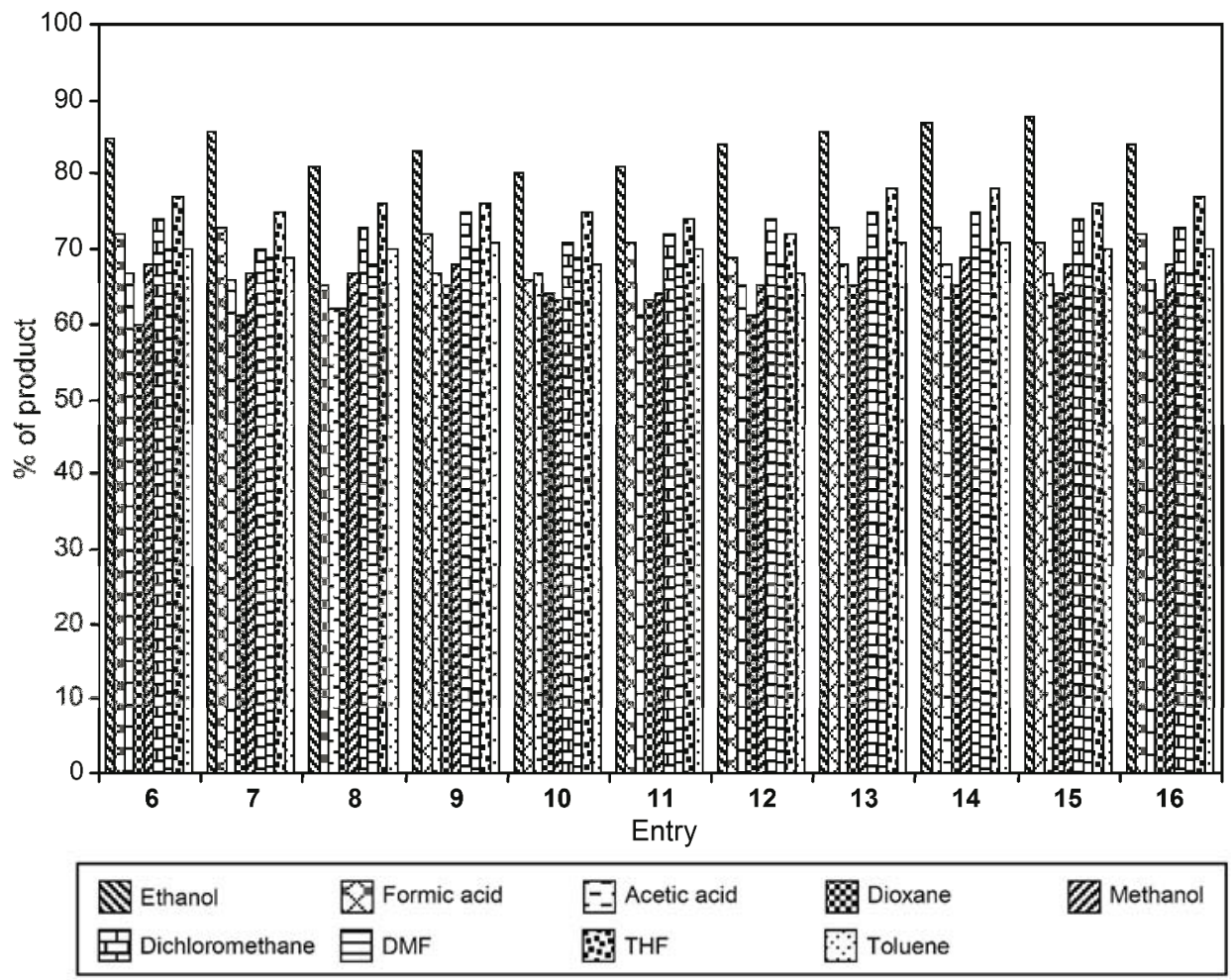

Fig. 1. The effect of solvents for synthesis of Schiff's bases by perchloric acid catalyzed condensation of aryl amines and benzaldehydes-clustered column chart (entries 6-16). 


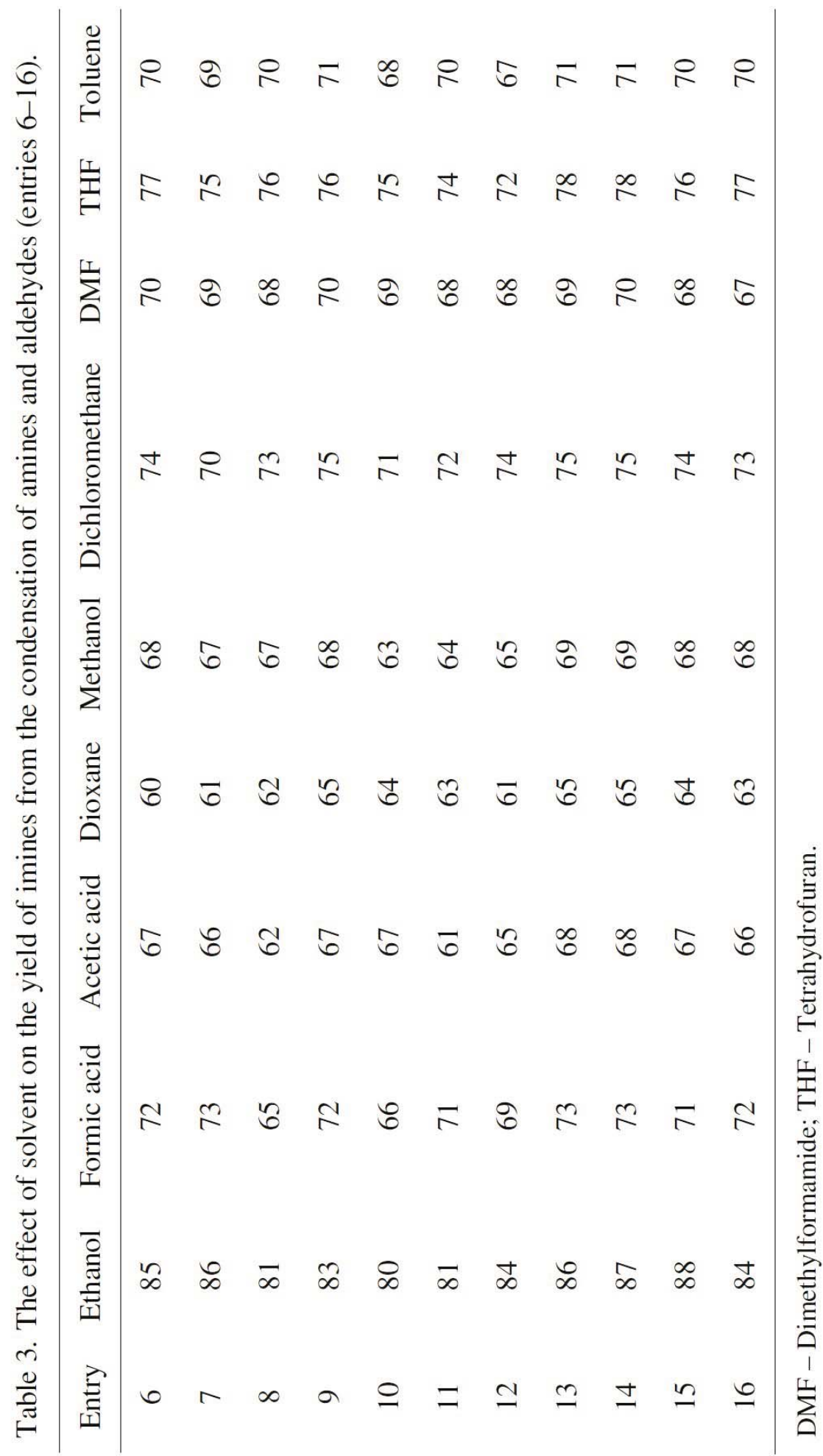




\subsection{Antibacterial activities}

The antibacterial screening effect of synthesized Schiff's bases are shown in Fig. 2(A-E).

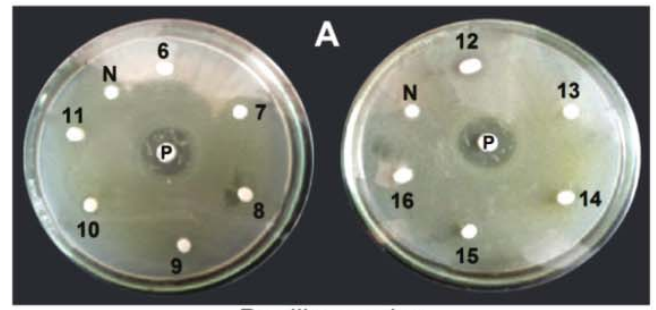

Bacillus cerius

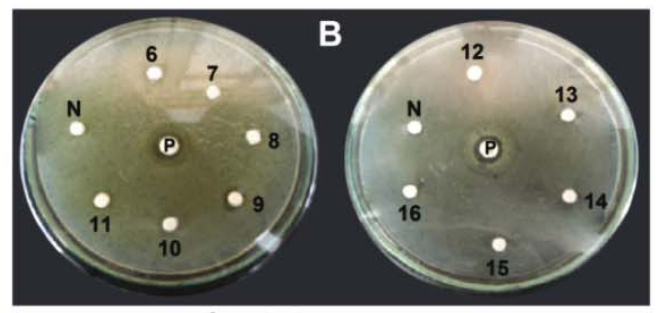

Staphylococcus aureus

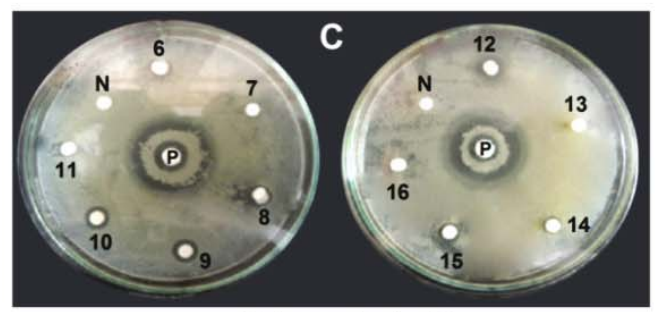

Salmonella typhi

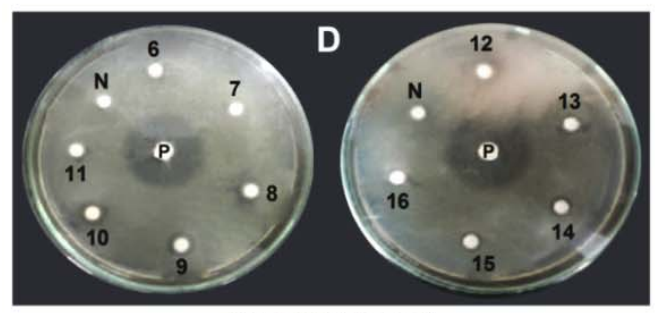

Escherichia coli

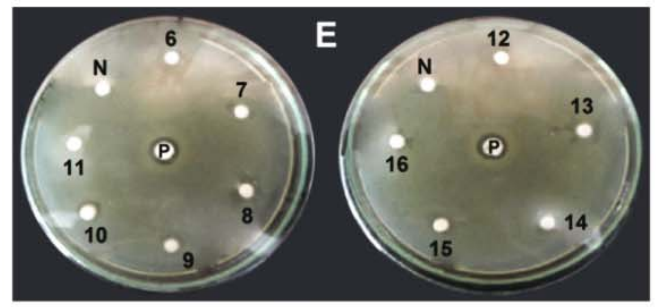

Klebsiella pneumoniae
Fig. 2(A-E). Antibacterial activities of imines petri-dishes (entries 6-16). N - Control (DMSO); P - Standard drug (Ampicillin). 
The antibacterial activities of all the synthesized imines have been studied against three Gram-positive pathogenic strains Bacillus cereus, Staphylococcus aureus, Salmonella typhi and two Gram-negative strains Escherichia coli and Klebsiella pneumoniae species. The disc diffusion technique was followed using the Bauer-Kirby [28] method, at a concentration of $250 \mu \mathrm{g} / \mathrm{cm}^{3}$ with Ampicillin used as the standard. The zone of inhibition is compared using Table 4 and the corresponding clustered column chart is shown in Fig. 3. A good antibacterial activity has been possessed by all compounds on the microorganisms in general. The imines having substituents $\mathrm{H}, 3-\mathrm{Cl}, 4-\mathrm{N}\left(\mathrm{CH}_{3}\right)_{2}, 4-\mathrm{F}$ and $4-\mathrm{OCH}_{3}$ showed good activity against $B$. cereus strains. The $\mathrm{H}, 3-\mathrm{Br}, 4-\mathrm{N}\left(\mathrm{CH}_{3}\right)_{2}$, 4-F, 4- $\mathrm{OCH}_{3}, 4-\mathrm{CH}_{3}$ and $3-\mathrm{NO}_{2}$ substituted imines shows good activity against $S$. aureus strains. All imines showed good antibacterial activity against $S$. typhi except $3-\mathrm{OCH}_{3}$ and $4-\mathrm{Cl}$ substituted imines. Similarly all compounds showed good activities on $E$. coli and $K$. pneumoniae species except $4-\mathrm{Cl}$ and $4-\mathrm{CH}_{3}$ substituted imines. DMSO was not produced any zone inhibition against the bacterial strains tested.

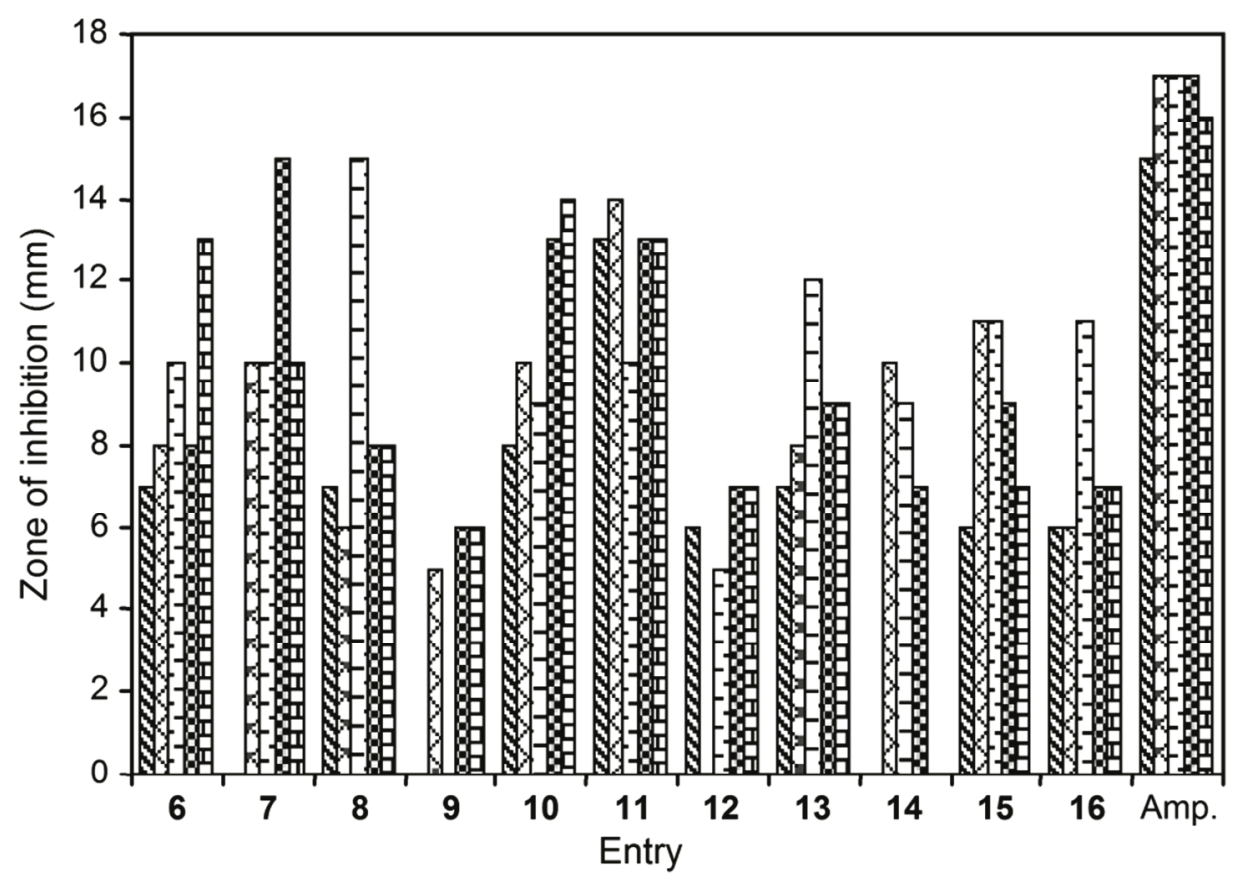

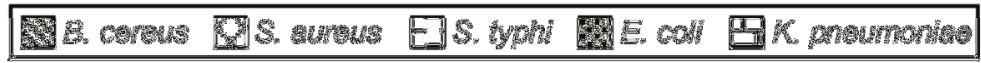

Amp. = Ampicillin (statandard drug)

Fig. 3. Antibacterial activities of imines clustered column chart (entries 6-16). 


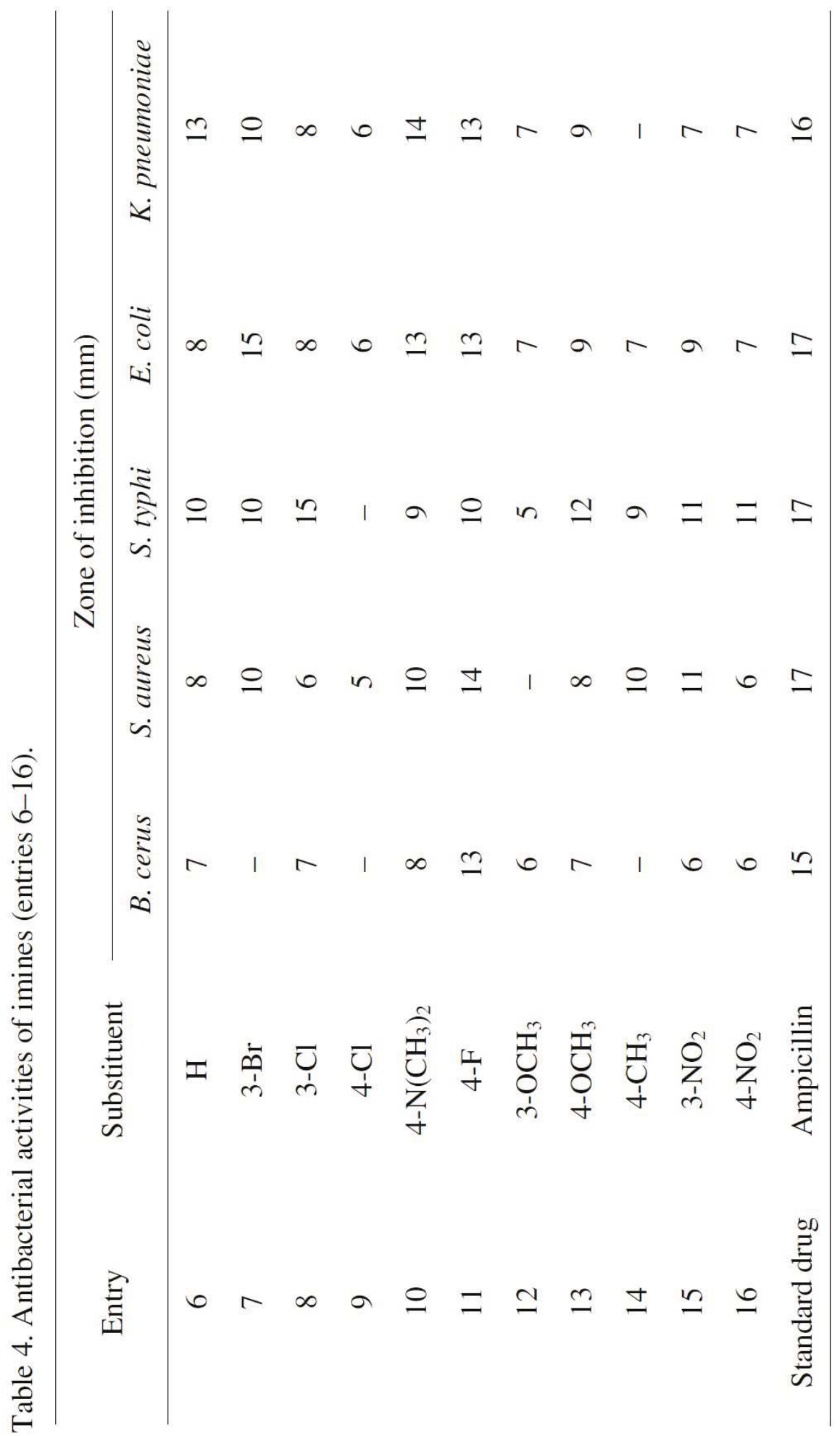




\section{CONCLUSIONS}

A series of aryl imines have been synthesized by condensation of aryl amines and substituted benzaldehydes using perchloric acid under the reflux condition. This reaction protocol offers a simple, easier work-up procedure and high yield. These imines were characterized by their physical constants, spectral data. The UV, IR, NMR spectral data of these imines has been correlated with Hammett substituent constants, F and R parameters. From the results of statistical analyses, the effects of the substituents on the spectral data have been studied. The antibacterial activities of all synthesized imines have been studied using Bauer-Kirby method.

\section{ACKNOWLEDGEMENT}

The authors thank DST NMR Facility Unit, Department of Chemistry, Annamalai University, Annamalainagar 608002 for recording NMR spectra.

\section{REFERENCES}

[1] K.Y. Lau, A. Mayr, K.K. Cheung, Inorg. Chim. Acta, 285, 223, (1999).

[2] A.S. Shawali, N.M.S. Harb, K.O. Badahdah, J. Heterocycl. Chem., 22, 1397, (1985).

[3] S. France, M.H. Shah, A. Weatherwax, H. Wack, J.P. Roth, T. Lectka, J. Am. Chem. Soc., 127, 1206, (2005).

[4] C.K.Z. Andrade, S.C.S. Takeda, L.M. Alves, J.P. Rodrigues, P.A.Z. Suarez, R.F. Brandao, V.C.D. Soares, Synlett, 12, 2135, (2004).

[5] M. Abid, M. Savolainen, S. Landge, J. Hu, G.K. Surya Prakash, G.A. Olah, B. Torok, J. Fluorine Chem., 128, 587, (2007).

[6] D.A. Barr, G. Donegn, R. Grigg, J. Chem. Soc. Perkin Trans. 1, 1550, (1989).

[7] L. Blackburn, R.J.K. Taylor, Org. Lett., 3, 1637, (2001).

[8] M. Gopalakrishnan, P. Sureshkumar, V. Kanagarajan, J. Thanusu, J. Res. Chem. Intermed., 33, 541, (2007). 
[9] R.T. McBurney, F. Portela-Cubillo, J.C. Walton, RSC Adv., 2, 1264, (2012).

[10] A.K. Chakraborti, S. Bhagat, S. Rudrawar, Tetrahedron Lett., 45, 7641, (2004).

[11] R.S. Varma, Green Chem., 1, 43, (1999).

[12] A. Hasaninejad, A. Zare, H. Sharghi, M. Shekouhy, Arkivoc, 11, 64, (2008).

[13] C.M. Bode, A. Ting, S.E. Schaus, Tetrahedron, 62, 11499, (2006).

[14] M.A. Vazquez, M. Landa, L. Reyes, M.J. Tamariz, D. Francisco, Synth. Commun., 34, 2705, (2004).

[15] M. Hudlicky, Oxidations in Organic Chemistry, ACS Monograph Series 186, American Chemical Society, Washington DC, (1990), p. 174.

[16] S.M. Landge, V. Atanassova, M. Thimmaiah, B. Torok, Tetrahedron Lett., 48, 5161, (2007).

[17] A. Kundu, N.A. Shakil, D.B. Saxena, J. Pankaj Kumar, S. Walia, J. Environ. Sci. Health B, 44, 428, (2009).

[18] S.F. Martin, Pure Appl. Chem., 81, 195, (2009).

[19] S.E. Denmark, G.L. Beutner, Angew. Chem. Int. Ed., 47, 1560, (2008).

[20] P.K. Kabada, J. Heterocycl. Chem., 12, 143, (1975).

[21] P.K. Kabada, J. Med. Chem., 31, 196, (1998).

[22] D. Bandyopadhyay, S. Mukherjee, R.R. Rodriguez, B.K. Banik, Molecules, 15, 4207, (2010).

[23] V. Tiwari, J. Meshram, P. Ali, Der. Pharm. Chem., 2, 187, (2010).

[24] M. Sayyed, S. Mokle, M. Bokhare, A. Mankar, S. Surwase, S. Bhusare, Y. Vibhute, Arkivoc, 2, 187, (2006).

[25] A.A. Bakibaev, V.K. Gorshkova, O.V. Arbit, V.D. Filimonov, A.S. Saratikov, Pharm. Chem. J., 28, 335, (1994).

[26] S. Kantevari, T. Yempala, P. Yogeeswari, D. Sriram, B. Sridhar, Bioorg. Med. Chem. Lett., 21, 4316, (2011).

[27] R. Yadav, S.D. Srivastava, S.K. Srivastava, Indian J. Chem., 44B, 1262, (2005).

[28] B.W. Bauer, W.M.M. Kirby, J.C. Sherris, M. Truck, Am. J. Clin. Pathol., 45, 493, (1966).

[29] R. Suresh, D. Kamalakkannan, K. Ranganathan, R. Arulkumaran, R. Sundararajan, S.P. Sakthinathan, S. Vijayakumar, K. Sathiyamoorthi, V. Mala, G. Vanangamudi, K. Thirumurthy, P. Mayavel, G. Thirunarayanan, Spectrochim. Acta A, 101, 239, (2013). 